\title{
Automatic 3D Pattern Making of Fitted Upper Garments Considering Individuals' Figures and Shear Limit of Fabrics
}

\author{
Yo TAKAHASHI*, Akito SOFUE*, KyoungOk KIM** and Masayuki TAKATERA*** \\ * Division of Science and Technology, Graduate School of Shinshu University, 3-15-1 Tokida, Ueda, Nagano 386-8567, Japan \\ ** Interdisciplinary Graduate School of Science and Technology, Shinshu University, 3-15-1 Tokida, Ueda, Nagano 386-8567, Japan \\ *** Faculty of Textile Science and Technology, Shinshu University, 3-15-1 Tokida, Ueda, Nagano 386-8567, Japan
}

\begin{abstract}
This study aims to develop a method for computerized pattern manufacturing system of basic upper garments which are fitted for individuals. We developed a method of individual pattern making for upper garments by modifying a traditional draping system. The required shear limit of the fabric to cover a body varies according to the figure of the wearer. We manufactured upper garment patterns for 8 subjects using our pattern making system. The large shear limit $\left(64^{\circ}\right)$ or the ordinarily shear limit $\left(32^{\circ}\right)$ were set to consider the influence of the shear deformation for a fabric. The grain lines and cutting lines were set at the same position as the traditional basic pattern. It was found that it is necessary to consider shear limit and to examine positions of the grain line and cutting line for individual. Furthermore, by setting the positions of the grain line and cutting line for individuals, it was able to make upper garments which were fitted for individuals.
\end{abstract}

Keywords: Individual pattern making, Upper garment, Ease allowance, 3D human body shape, shear limit

\section{INTRODUCTION}

Garment is the most important product reflecting individual kansei. Production of garment in accord with individual preference is a subject of the kansei engineering. At first it is necessary to establish a design method of the garments which adapted individual figures to solve the subject. Most garments in the market are ready-made clothes today. Consumers choose clothing to fits themselves among the products and purchase it. However, the kinds of size are limited in ready-made clothes, and most people cannot wear clothes being the fittest state.

On the other hand, draping and basting are used to make clothing which reflects an individual figure. Draping is a technique to get a pattern by cutting and assembling cloth as putting cloth on the surface of a body considering fabric properties. In the draping, a dummy model which imitates a person of a standard figure and measurements is generally used. When the measurement of an existing dummy is different from that of the customer's figure, correction will be performed with putting some padding on the dummy. However, it is difficult to reproduce figures of customers completely by it. In addition, a lot of measurement reference points exist in body surface. Moreover, high skill and long measuring time are necessary in order to measure the human body precisely.
By diffusion of three dimensional measuring instruments in recent years, we can measure human whole-body precisely during a short time and without high skill. In addition, by the development of computer technology, CAD system of modeling for the human body in virtual space, simulation of wearing clothing, and pattern making in a three dimensional space which utilized the virtual body model have been developed [1]. Furthermore, many studies of computer aided pattern making method have been also performed [2-7]. However, these systems can make limited kinds of the clothing and they did not actually confirm the systems by making clothing. Therefore, detailed examination and verification of each clothes type are necessary for practical uses.

In our previous study, we have developed a system of individualized pattern making by modifying a traditional draping technique. The skirt and trousers were successfully made with the system [8-12]. A method of individual pattern making for the upper garment pattern was also proposed in our previous study [13].

In this study, we aim to develop a design method for computerized pattern manufacturing of basic upper garments for an individual's unique body considering shear limit of fabrics. The relationship between clothing patterns and fabric property such as shear properties will be necessary to be considered to make garments more reflected individual preference. Woven fabrics can form curved surface because of shear property. Kumoda et al. [14] 
investigated the shear limit of fabrics and they clarified that the shear limit depends on fabrics. The positions of the grain line and cutting line for pattern will be also affected by an individual figure and fabric properties. These properties have not been considered in the computer aided pattern making method yet. Therefore, the shear limit of a fabric, the positions of the grain line and cutting line for individuals were considered in the system. The system was verified with the real human body scan data. We obtained the individual patterns with individually reconstructed $3 \mathrm{D}$ body shapes by computerized pattern development.

Three dimensional shape data of a body were acquired by a three dimensional scanner and a body model was constructed in virtual space with the data. The scanned data were converted to polyline model. The polyline model is a human body model which consists of contour lines of equal vertical coordinate of the human body. The clothing model was constructed by controlling of the polyline model. We made a basic clothing model from the human body model. By this clothing model, we were able to make clothing pattern for different form of shoulder, bust, back and waist which were optimized for an individual.

The constructing method of 3D upper body shape for an individual's unique body with ease allowance was made by the sweep method, unevenness expansion, and the convex hull. In our system, complex curved lines such as armhole $(\mathrm{AH})$ curve, a neck line and the dart lines on upper garments pattern were able to be created automatically. The armhole curve was made by cutting by a plane defined by points of the sleeve cap top and the lowest armpit point on 3D upper body shape.

The sleeve part for the individual's unique body was produced by making the sweep of a polygon model parallelized to a shoulder line. Then the sleeve model was made by a sweep of armhole at 45 degrees (the angle from the horizontal plane which assumed the center of $\mathrm{AH})$. The polyline model was converted into a triangular polygon mesh model before the draping process.

In draping of one piece of woven fabric model, one set of grainline, a warp and a weft, is set on the clothing model first. The fabric model is expressed with the lattice of regular intervals. The fabric model covers the surface from the grainlines while making shear [9]. The covering stops at the edge of fabric model or the shear limit. The shear limit is a limit shear angle which fabric can shear without a wrinkle. See Figure 1. Incorrect setting of shear limit will causes unpleasant wrinkles and poor fit. After the fabric model is fitted to the surface, panels can then be
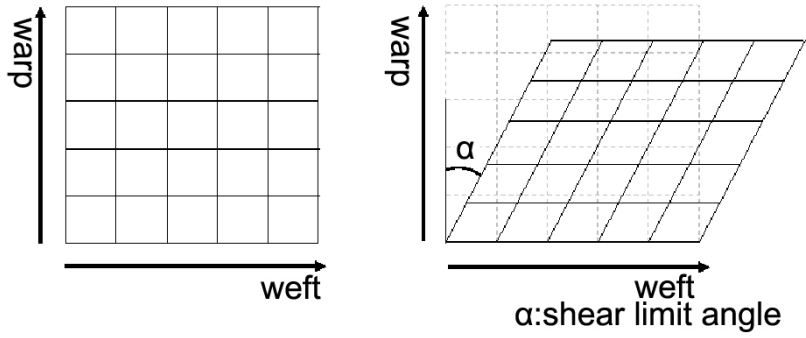

Figure 1: Shear limit deformation of fabric model.

cut by planes or curved lines. Patterns are obtained by making angle of fabric lattices at right angles from 3D contoured panels into $2 \mathrm{D}$. In this process, 3D cutting line is flattened on the 2D pattern. Finally we can archive the pattern [9].

\section{EXPERIMENT}

The validity of our system was investigated by making upper garments of 8 subjects. Three-dimensional data of 8 subjects were obtained with the clothing of Figure 2 by 3D scanner VOXELAN (HAMANO ENGINEERING CO., LTD.) as shown in Figure 3.

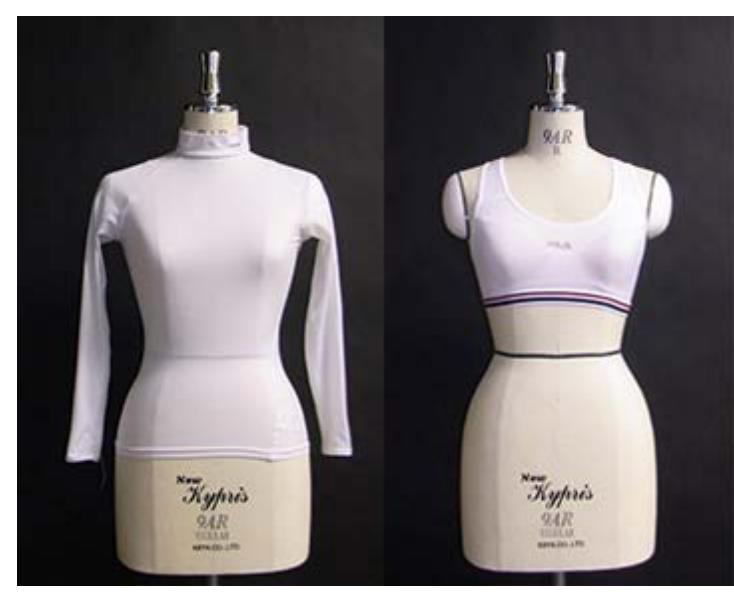

Figure 2: Clothing for 3D scan. (a) For Subject 1 - 4 (b) For Subject 5 - 8
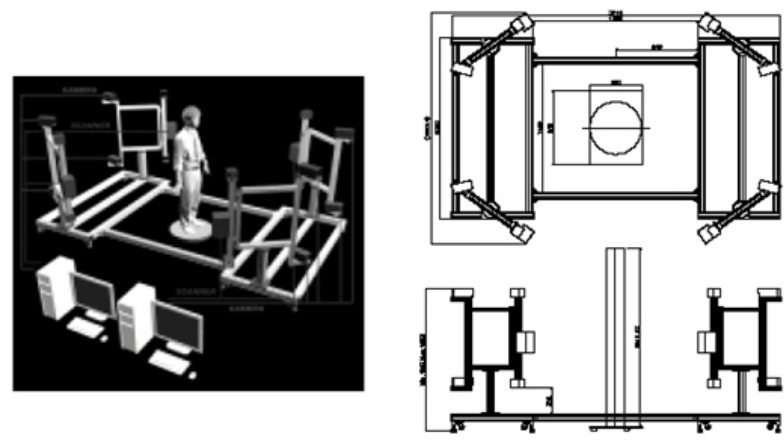

Figure 3: Three dimensional scanner. 
Table 1 shows the measurements of the subjects. We made upper garment patterns using 3D scanned body data of the subjects. We used glainline and cutting line of a traditional basic pattern (Bunka-style, see Figure 4) [15]. The shear limit were set to large $\left(64^{\circ}\right)$ and ordinarily $\left(32^{\circ}\right)$. Kumoda et al [14] investigated the shear limit of a fabric and suggested that ordinarily shear limits is between 26 and $32^{\circ}$. Therefore, we considered ordinarily $\left(32^{\circ}\right)$ shear limits and large $\left(64^{\circ}\right)$ shear limits.

At first, we manufactured patterns with large $\left(64^{\circ}\right)$ shear limits. We made tight fit clothes models from the polyline body model of subjects. We cut unnecessary polyline of the initial model. The dent of the bust and the shoulder blade were reconstructed by convex hull. A tight fit clothes model is shown in Figure 5-(a). In addition, we performed the trunk manipulation of the poly lines to make a semi-tight-fit model. Figure 5-(b) shows a semitight-fit model.

Table 1: Subject measurement.

\begin{tabular}{|c|c|c|}
\hline SUBJECT & BUST $(\mathrm{cm})$ & WAIST $(\mathrm{cm})$ \\
\hline 1 & 80.6 & 65.1 \\
\hline 2 & 95.1 & 77.1 \\
\hline 3 & 89.0 & 69.1 \\
\hline 4 & 80.6 & 68.5 \\
\hline 5 & 89.6 & 70.4 \\
\hline 6 & 84.9 & 67.1 \\
\hline 7 & 88.5 & 73.2 \\
\hline 8 & 79.7 & 61.5 \\
\hline
\end{tabular}

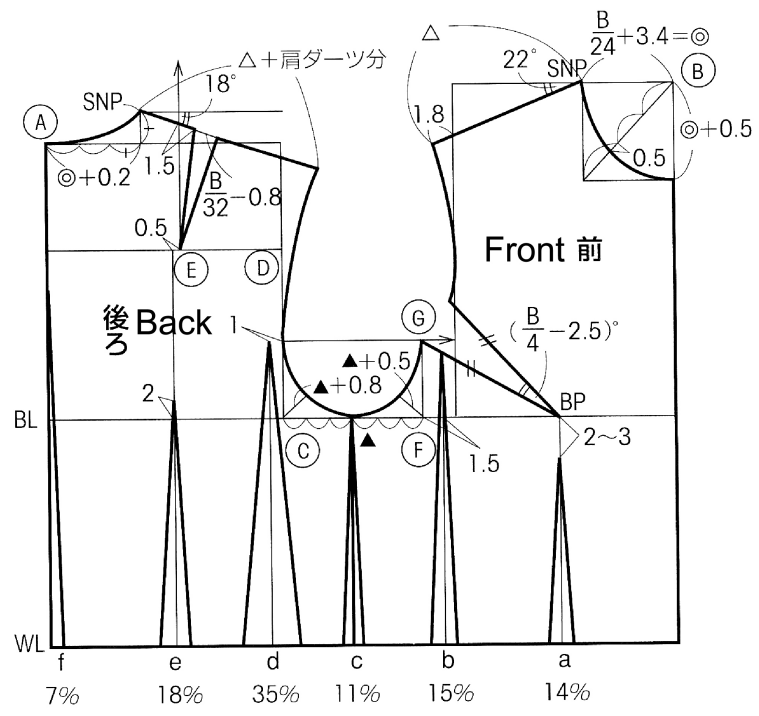

Figure 4: Bunka-style basic pattern [15].
We produced the sleeve part for a clothes model by sweep of the outline for the armhole $(\mathrm{AH})$ parallelized to the shoulder line. The sleeve model was made by sweep of $\mathrm{AH}$ at 45 degrees as shown in Figure 6.

The grain lines and cutting lines were set in the similar position as the traditional basic pattern (Bunka-style) depending on the subject figure as shown in Figure 7. We also set the warp grain line with a shoulder line perpendicularly and the weft grain line in parallel with a shoulder line as shown in Figure 8.

In addition to setting large shear limit $\left(64^{\circ}\right)$, we manufactured a pattern with ordinarily $\left(32^{\circ}\right)$ shear limits. Using an obtained pattern, we made an upper garment with a plain fabric (Table 2). The fit and wrinkle of the manufactured garments were investigated when the subject wore it.

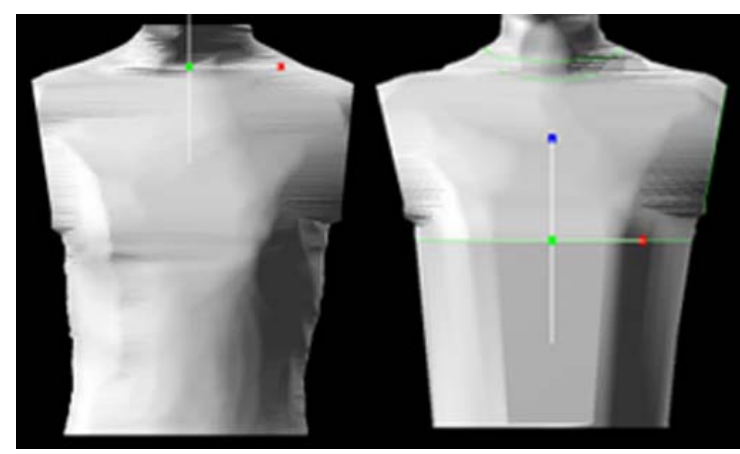

(a)

(b)

Figure 5: (a) Tight-fit model (b) Semi-tight-fit model.

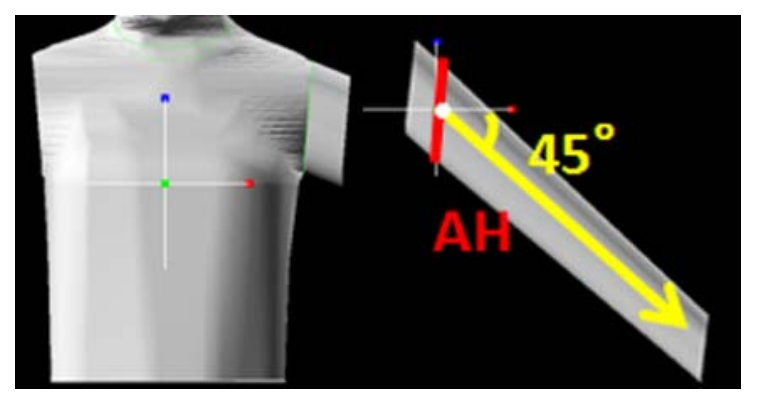

(a)

(b)

Figure 6: (a) Semi-tight-fit model with sleeve part (b) Sleeve model.

Table 2: Material for experimental garments.

\begin{tabular}{|c|c|c|c|}
\hline Material & Structure & $\begin{array}{c}\text { Weave density } \\
(/ \mathrm{cm})\end{array}$ & $\begin{array}{c}\text { Yarn count } \\
(\mathrm{tex})\end{array}$ \\
\hline cotton $100 \%$ & Plain & $\begin{array}{c}\text { warp30 } \\
\text { weft30 }\end{array}$ & $\begin{array}{c}\text { warp28 } \\
\text { weft 36 }\end{array}$ \\
\hline
\end{tabular}




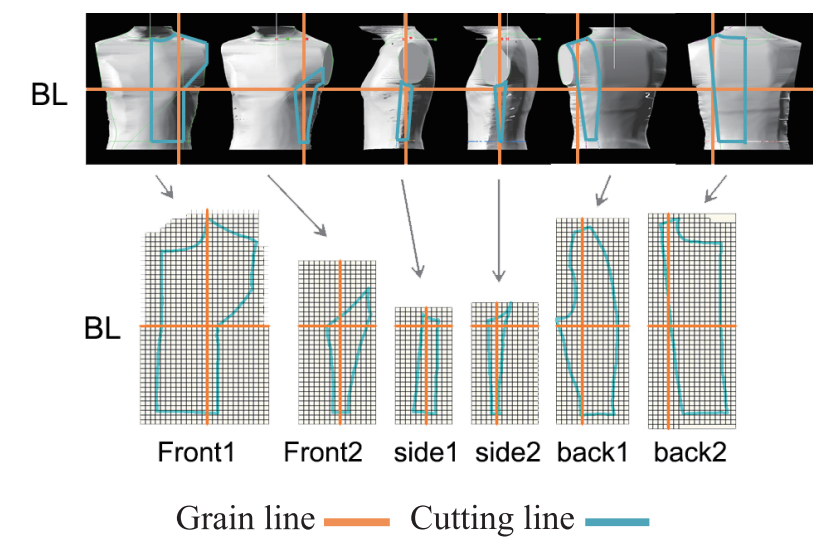

Figure 7: Setting of traditional grain line and cutting line and obtained pattern.

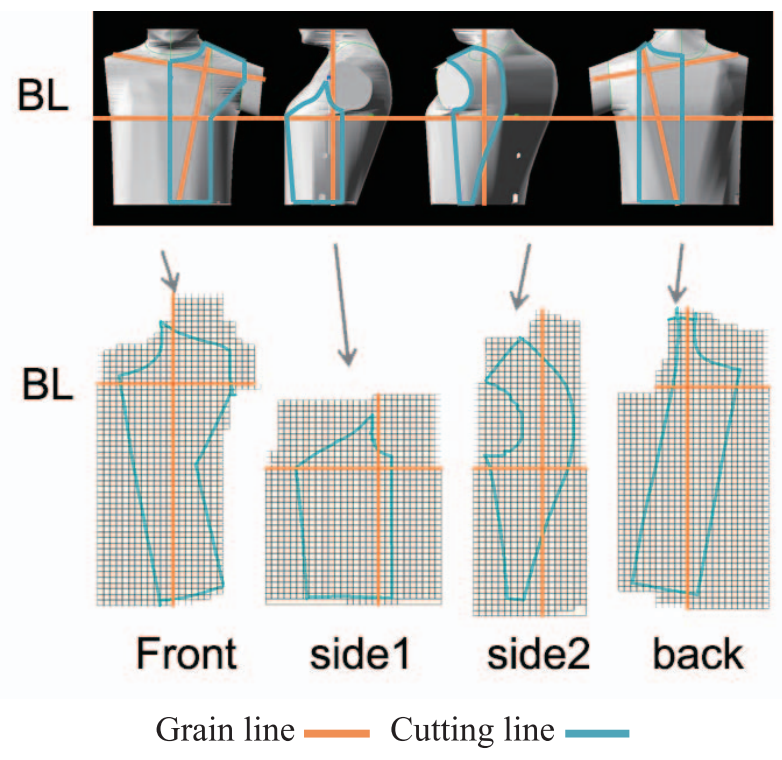

Figure 8: Setting of traditional grain line and cutting line and obtained pattern.

\section{RESULTS AND DISCUSSION}

The patterns for each subject were able to be made by proposed method as shown in Figures 9 and 11. Figure 9 shows the patterns and garments with large shear limits. With manufactured patterns considering large shear limits, we made the tight-fit upper garments for subject 1 and 2 . For subject 1, we made two types of tight fit patterns which are consisted of 6 parts and 4 parts. The sleeve was also able to be produced by making the sweep of a polygon model of $\mathrm{AH}$ at 45 degrees as shown in Figure 6.

It was able to make upper garments for each subject with obtained patterns. However, subject 2 got a difference of the long length to cutting line of the shoulder as shown in Figure 9 so it was hard to sew. Bust size of subject 2 is relatively larger than the other subjects. When the bust size is large, the required deformation of the

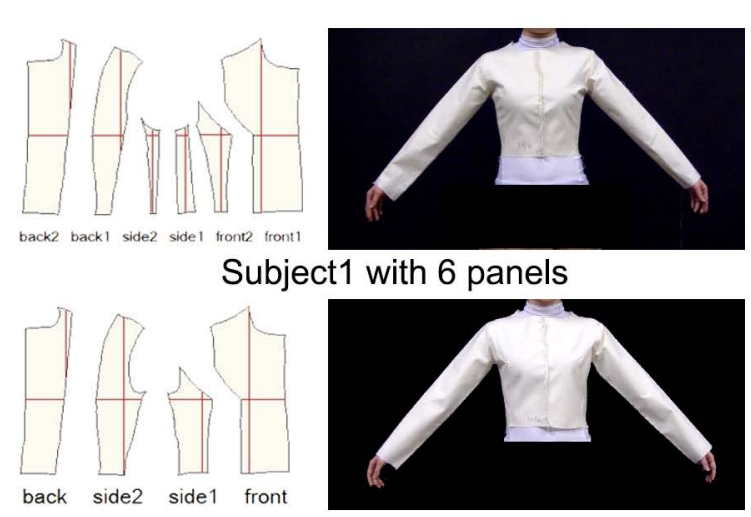

Subject 1 with 4 panels
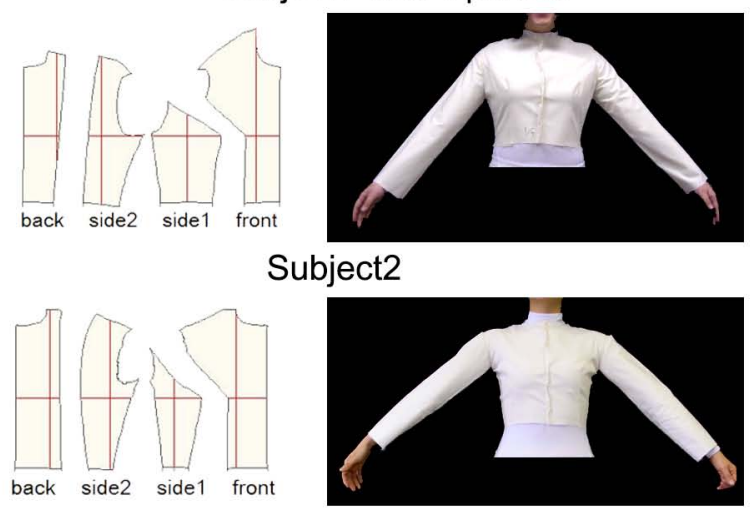

Subject3

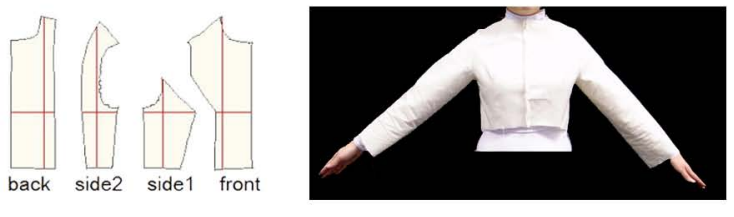

Subject4

Figure 9: Pattern made with large shear limit and manufactured upper garment.

fabric will be large so that it was hard to sew. Though it was able to make an upper garment for subject 2, superfluous wrinkles occurred by a difference of the length of the shoulder cutting lines while wearing on it.

In addition, we made semi-tight-fit pattern and produced upper garment as the procedure of tight-fit by trunk manipulation of the poly line for subject 3 and 4 . Cutting line was positioned at the similar place with Figure 8. Even though it was able to make upper garments for subject 3 and 4, those had similar difficulty with subject 2 when sewing. The reason was considered as the shear limits. This is because the shear limit of the used woven fabric is smaller than the maximum shear angle in the cover process.

The investigated shear limits for each manufactured panels in the cover process are shown in tables 3 and 4 . In the case of setting grain line and cutting line with the same position as the traditional basic pattern as Figure 7, necessary shear limits were mostly larger than ones of 
Table 3: Measured maximum shear for each part of pattern made with large shear limit.

\begin{tabular}{|c|c|c|c|c|}
\hline Subject & Front & Side 1 & Side 2 & Back \\
\hline 1 & $21^{\circ}$ & $20^{\circ}$ & $48^{\circ}$ & $45^{\circ}$ \\
\hline 2 & $40^{\circ}$ & $15^{\circ}$ & $52^{\circ}$ & $20^{\circ}$ \\
\hline 3 & $31^{\circ}$ & $34^{\circ}$ & $46^{\circ}$ & $40^{\circ}$ \\
\hline 4 & $25^{\circ}$ & $10^{\circ}$ & $48^{\circ}$ & $30^{\circ}$ \\
\hline
\end{tabular}

subject 1 as Figure 6. Among the shear limits of each part, shear limit of side 2 were larger than ones of the other parts. Especially, it was necessary to consider the shear limit as $52^{\circ}$ for side 2 panel of the subject 2 . Therefore, it was clear that a fabric which is easy to give larger shear is required for the panel of side 2 and back to make such pattern of cutting line as Figure 8. Otherwise it is necessary to set the grain lines and cutting lines to cover with smaller shear limit.

Furthermore, the subjects were hard to wear the garments with large shear limit $\left(64^{\circ}\right)$ and showed unpleasant tight feeling while wearing them. Clothing patterns unconsidered the fabric properties cause unpleasant wrinkle and uncomfortable which will be against to the individual preference.

Figure 11 shows the patterns and garments with ordinary shear limits. For the semi-tight fit patterns, we set the warp grain line with a shoulder line perpendicularly and the weft grain line in parallel with a shoulder line. The maximum shear angle in the sleeve cap top area became smaller and the fabric model was able to cover it. We set the cutting line to the border of the fitting range as shown in Figure 10 and manufactured a pattern. In the case of subject 7, we had to let a cutting line go to $\mathrm{AH}$ because the scapular undulation was small. Accordingly the shapes of panels were different from other subjects as shown in Figure 11.

Therefore, it was necessary to examine the positions of the grain line and cutting line for individuals. In our experiment, we found a new grain line position which maximum shear angle becomes smaller and set a new cutting line to the part that shear angles of the covering fabric became large.

The upper garments with the patterns by ordinary $\left(32^{\circ}\right)$ shear limit were easy for sewing and showed no unusual wrinkles. We were able to make a pattern even the shear limit was $32^{\circ}$ when we used the grain lines and the position of cutting lines which we proposed. The panels of front and side 2 were needed to set larger shear limits than ones of the other parts. Even though it was able to make upper garments with the patterns set large shear limit $\left(64^{\circ}\right)$, those had difficulty when forming. With the patterns by ordinary shear limit $\left(32^{\circ}\right)$ considered fabric properties, the upper garments were easy for sewing and showed no unusual wrinkles.

While wearing garments with ordinary shear limit $\left(32^{\circ}\right)$, the subjects were easy to wear them and showed comfortable feeling. Therefore, it became clear that more prefer garments for individuals can be made by considering shear properties of fabric.

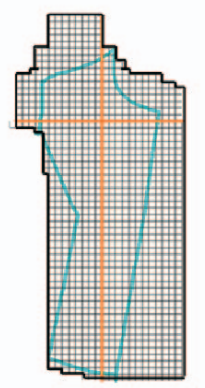

Grain line $=$ Cutting line

The border of the fitting range

Figure 10: Front panel of the pattern.

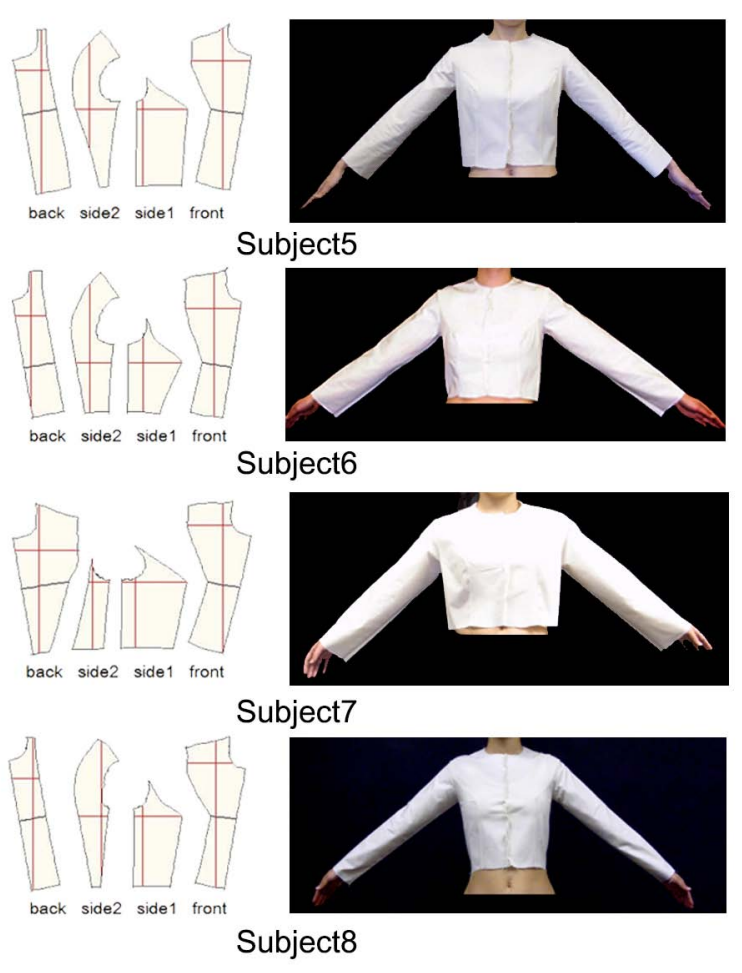

Figure 11: Pattern made with ordinary shear limit and manufactured upper garment.

Table 4: Measured maximum shear for each part of pattern made with large shear limit.

\begin{tabular}{|c|c|c|c|c|}
\hline Subject & Front & Side 1 & Side 2 & Back \\
\hline 5 & $24^{\circ}$ & $15^{\circ}$ & $31^{\circ}$ & $20^{\circ}$ \\
\hline 6 & $23^{\circ}$ & $17^{\circ}$ & $29^{\circ}$ & $13^{\circ}$ \\
\hline 7 & $21^{\circ}$ & $10^{\circ}$ & $32^{\circ}$ & $18^{\circ}$ \\
\hline 8 & $25^{\circ}$ & $20^{\circ}$ & $32^{\circ}$ & $13^{\circ}$ \\
\hline
\end{tabular}




\section{CONCLUSION}

We developed a computerized method of individualized pattern making for upper garments by modifying a traditional draping method. Shear limit of a fabric and the position of the grain line and cutting line were considered to adjust the pattern to individual figures. It was able to make upper garments which were fitted for individuals by proposed method. With the patterns of large shear limit, the garments were hard to sew and showed unpleasant wrinkle. The garments with patterns of ordinary shear limit were easier to sew than garments with large shear limit and showed no unusual wrinkles. Those were easy to wear and fitted the subjects' preferences. Therefore, it became clear the necessity of using a shear limit of the practical fabric. The required shear limit depends on the individual figure. We can make the required shear limit small by devising the positions of the grain lines and cutting lines. Therefore, it is found that it is necessary to consider the shear limits and to examine the positions of the grain lines and cutting lines to make more fitted garments for individuals. If it is possible to place the ease allowance in the clothing model effectively with the manufactured patterns by our method, the garments reflected individual preference and fitness can be made by our system.

\section{ACKNOWLEDGEMENTS}

This work was supported by JSPS KAKENHI Grant number 24220012.

\section{REFERENCES}

1. i-Designer, http://www.i-designer-web.com/ [Accessed 2012 Oct. 30],

DressingSim LSX, http://www.digitalfashion.jp/new/ product/dressingsim_lsx/index.html [Accessed 2012 Oct. 30].

2. HAOREBA®, http://www.dressingsim.com/new/ product/haoreba/index.html [Accessed 2012 Oct. 30].

3. Hitoto, http://www.toray-acs.com/ [Accessed 2012 Oct. 30].

4. Bigliani R, Eischen J.W, House D.H and Breen D.E: Collision detection in cloth modeling in cloth modeling and animation. A.K. Peters, Ltd., pp.199-205 (2000).
5. Heisey. F, Brown. P and Johnson. R.F: Threedimensional pattern drafting - Part 1: projection, Textile Research Journal, 60(11), pp.690-696 (1990).

6. Heisey. F, Brown. P and Johnson. R.F: Threedimensional pattern drafting - Part 2: garment modeling, Textile Research Journal, 60(12), pp.731737 (1990).

7. Kim S, and Park C.K: Basic garment pattern generation using geometric modeling method, International Journal of Clothing Science and Technology, 19(1), pp.7-17 (2007).

8. Youngsook Cho, Takuya Komatsu, Masayuki Takatera, Shigeru Inui, and Yoshio Shimizu: Posture and depth adjustable 3D body model for individual pattern making, International Journal of Clothing Science \& Technology, 18(2), pp.96-107 (2006).

9. Youngsook Cho, Takuya Komatsu, Hyejun Park, Shigeru Inui, Masayuki Takatera, and Yoshio Shimizu: Individual pattern making using computerized draping method for clothing, Textile Research Journal, 76(8), pp.646-665 (2006).

10. Youngsook Cho, Masayuki Takatera, Keiichi Tsuchiya, Shigeru Inui, Hyejun Park, and Yoshio Shimizu: Computerized pattern making focus on fitting to 3D human body shape, International Journal of Clothing Science and Technology, 22(1), pp.16-24 (2010).

11. Keiichi Tsuchiya, Youngsook Cho, and Masayuki Takatera: Individual Pattern Making of Skirts and Pants Using Computerized Draping Method, In Proc. KEER (2007).

12. Masayuki Takatera, Keiichi Tsuchiya, KyoungOk Kim, and Youngsook Cho: Computer Aided Design of Individualized Best Fitting Pants, In Proc. KEER (2010).

13. Akito SOFUE, Masayuki TAKATERA, Yoshiko TAKAHASHI, and KyoungOk KIM: Construction of upper garment model for computerized draping method from 3D human body model, 6th Japan Society of Kansei Engineering Spring Conference, CD-ROM, 24D-01 (2011).

14. Naoko Kumoda, Masami Hiraide, Masayuki Takatera, and Yoshio Shimizu: Evaluation of heat set effect of woven fabrics under bias direction load, Japan. Res. Assn. Text. End-Uses, 42(1), pp.51-59 (2001).

15. Machiko Miyoshi: Fukusou Zoukeigaku Riron Hen. Bunka Women's University, Japan. 


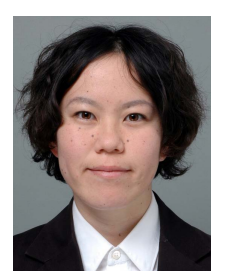

\section{Yo TAKAHASHI}

Yo Takahashi is a graduate student in master course at Division of Science and Technology, Shinshu University, Japan. Her interests are Kansei engineering, textile engineering and clothing engineering.

\section{Akito SOFUE}

Akito Sofue received the M.E. degree from Division of Science and Technology, Graduate School of Shinshu University, Japan in 2011.

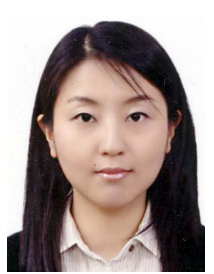

\section{KyoungOk KIM}

KyoungOk Kim is a graduate student in doctor course at Department of Bioscience and Textile technology, Shinshu University, Japan. Her interests are Kansei engineering, textile engineering and clothing engineering.

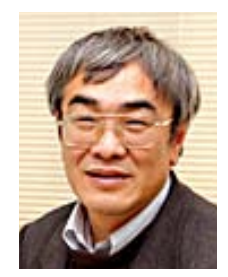

\section{Masayuki TAKATERA}

Masayuki Takatera is a Professor of Faculty of Textile Science and Technology, Shinshu University, Division of Textile and Kansei Engineering, Kansei Engineering Course, Japan. He is currently vice dean of JSKE. His interests are Kansei engineering, textile engineering and clothing engineering. 